\title{
The current situation of child and adolescent psychological problems and intervention in China
}

\author{
Wenyan Jiao', Lin Liü, Rui Lì ${ }^{3}$, Na Zhao ${ }^{3}$ \\ ${ }^{1}$ Department of Psychiatry, Shaanxi Provincial People's Hospital, Xi'an, 710068,P.R, ${ }^{2}$ School of Communication Engineering, Xi'an University \\ of Science and Technology Xi'an, China 710068, ${ }^{3}$ Children's Hospital, Shaanxi Provincial People's Hospital, Xi'an, 710068, P.R.
}

\section{A B S T R A C T}

In China, the psychological health problems of children and adolescents have been more and more serious recently. The psychological disorders not only have a lot of adverse effects for children and adolescents, but also were an important source of mental disease in adulthood. In order to make more people understand this serious problem, this paper summarized the current situation of child and adolescent mental health problems in China and the risk factors for child and adolescent mental health problems; additionally, the interventions of child and adolescent psychological problems were also reviewed in this paper.

\section{Access this article online}

Website:

http://nepjol.info/index.php/AJMS DOI: 10.3126/ajms.v7i3.13901 E-ISSN: 2091-0576 P-ISSN: 2467-9100

Key words: Child and adolescent, psypsychological, intervention, China

\section{INTRODUCTION}

After more than 20 years of reform and opening up, China has made great achievements in economy; the living standard of people has been greatly improved, but in the meantime, the psychological pressure of people is increasing drastically. Besides, emergence of psychological problems; the psychological health situation of youngsters and children is not optimistic in China recently. Consequently, to alleviate this, more attention must be paid on this aspect. In this review, we summarize the current situation of child and adolescent mental health problems and its systematic interventions.

The current situation of child and adolescent mental health problems in China

According to conservative estimates, ${ }^{1}$ there were 30 million minors suffering from various learning, emotion and mood disorders among 367 million minors whose age are under 18 in the Chinese mainland. The primary school students' psychological barriers are mainly including the problems of interpersonal relationship, emotional stability and learning adaptability, whose prevalence was 21.6 to $32.0 \%$. Among that, the prevalence of common ADHA (attention deficit hyperactivity disorder) in children was 1.70 to $5.07 \%$, of which $5.7 \%$ in Beijing, $6.0 \%$ in Hunan province. It is estimated that $30 \%$ of children ADHD would develop into adult attention deficit hyperactivity disorder. In addition, the prevalence of reading disorder was $2.9 \%$ in Beijing and $3.3 \%$ in Hunan province. Otherwise, there were 16.0 to $25.4 \%$ of college students to have a mental disorder, whose main performances included anxiety, terror, neurasthenia, force symptom and depression. ${ }^{1}$ Peking University institute of mental health have analyzed the reasons of dropping out for the college students of 16 different Beijing universities during ten years; and have found that the main reasons were infectious diseases before 1982, but mental disorders after $1982 .{ }^{2}$ Moreover, there will be a rising trend of psychological problems for college students; and the increases of neurosis behavior, such as anxiety, depression and so on, account for the dominated part. According to investigations, we have 30 million children and adolescents who suffered from all kinds of psychological problems; but we only have less than 150 doctors who worked on child psychiatry currently. The quantities of the U.S. population was one fifth of China, but the numbers of psychiatrists were 6000, much larger than China. ${ }^{3}$

This is a cruel and incredible scale. However, this is our current situation, it is the problem that we must overcome and find the best remedial measures. 
The risk factors for child and adolescent mental health problems

The modern medical mode has changed, from the physical model development to psychology model, and then evolved to social medical model. The occurrences and developments of all kinds of diseases were observed from these three aspects; adolescent mental health problems were no exception as well.

The occurrences of child and adolescent psychological problems were related to a lot of links in each developmental stage and many other factors. From the point of developmental stage, there are four periods involved, such as the pregnancy, perinatal stage, infancy and school-age periods; From the point of influence factors, the main factors were different in different periods. The stages of pregnancy and perinatal gave priority to biological factors, but the role of social psychological factors over ruled in infancy, especially in school going periods. Biological factors could constitute the quality basis of psychological barrier; nevertheless, environmental factors, education and social psychological factors constituted the inducement and adjustment factors of psychological barriers.

The impacts and roles of biological factors on disease were quite familiar, acceptable and understandable for most people. As many people know, a person could suffer from Down's syndrome, which showed silly looks and intelligent problems, when he had three $21^{\text {st }}$ chromosomes. In addition, if you suffered a head injury, you might have the symptoms of unconsciousness and memory loss; sometimes you could be very serious to the extent of the vegetative state. If the mother was short of folic acid during pregnancy, it could cause fetal nervous system development problems.

With the process of modernization and increasingly rapid pace of life, all kinds of competition and pressure is getting serious, psychological social factors play an important role in children and adolescents' mental health in such circumstance. The most pivotal thing is the domestic situation and parents' education approach of children's growth. The adverse factors can be listed as follow: Unharmonious relationship between parents, single-parent families and bad habits from family members; Moreover, emotional problems could not be ignored. There are two kinds of inappropriate emotion expression ways, ${ }^{4}$ one is overprotection or fondness. We can see a lot in our work that parents fulfill the demands of their children and are pampered. Parents think it shows their love for children but, this behavior deprives their children's opportunity to face with setbacks, stress and difficulties and weakens their ability of solving problems. The other improper way is to overlook the act of their children. Neglecting children's emotional needs and overlooking questions in their growth make children who have little experience for society have to solve problems all by themselves, this may have a negative effect on their growth. ${ }^{5}$

In addition, social and environmental factors also affect children's mental health inevitably. Chinese traditional culture advocate learning diligently, this spirits can be seen from a Chinese proverb: Ten thousands were a wooden bridge. Strict entrance requirements are bound to the growing learning pressure, many parents and teachers regard learning as the most important factor of success for children, thus ignore improving other abilities. It cannot be denied that National Policy of Family Planning play an important role in people's life. ${ }^{6}$ However, at least to some extent, it also makes children more self-centered, capricious and have weaker ability of interpersonal communication than predecessors. With the rapid improvement and increased material conditions from the influence of foreign culture, people's life style and values are also changing quietly, children pay more attention to pursuit money unconsciously.

Child and adolescent mental health problems is a public health problem

Child and adolescent mental health problem is a global public health problem, not belong to any hospital, any country or any culture. A global health psychology conference held by WHO in 1997 called on governments and institutions attached great importance to the psychological and behavioral problems and carried out various activities to serve all kinds of people. Among them, children are the key point of the mental health work service object. The WHO estimates that there are approximately five children and adolescents have emotional or behavioral problems before adult period worldwide. The problems can be listed as follow: Learning difficulties, lack of confidence, social impairment, smoking, alcoholism, drug abuse, pregnancy, running away from home, suicide, violence, crime, etc. only less than $1 / 5$ of the patients received treatment. ${ }^{7}$

Child and adolescent psychological problems and intervention

School-weary psychology: ${ }^{8}$ Some people think that family life (mainly evaluate the level of parent-child relationship), peer interaction (mainly assess the level of peer relationship) and school life (mainly estimate children's feeling in the school and whether they like school or not, etc.) have a close relationship with the appearance of weary.Compared with children who are normal in these three aspects, kids whose ability are deficiency in the evolution are likely to have weary mood. Child psychology help them establish self-identity and social role positioning and discuss related 
topics such as "what kind of person I want to be ", "how other people think of me". In order to establish healthy self-concept and set up correct outlook on life and world.

Depression of childhood: ${ }^{9}$ Pay attention to early recognition of depression of children. When a child is unhappy, lacks interest in the game, doesn't like to be alone, sleeps too little or too much, eats less food, shows lack of interest in classroom, has no interest in life and study. Other symptoms like memory murdered, low self-evaluation, headaches, abdominal pain, abdominal discomfort, dizziness, chest tightness, weakness. In some serious cases, the patients may have anorexia and suicide thoughts. Child depression need drug therapy, of course, also need psychological treatment. Psychological treatment play an important role in children's depression. ${ }^{10}$ This therapy includes supportive psychotherapy, behavior corrective therapy, cognitive therapy and family therapy. If the doctor isn't a professional psychological doctor, he can also play a role in treatment, finding children's confusion, doubt, fear, anxiety, temper, impulse and pain, giving them full of respect, understanding, compassionate, and finally encouraging them to cooperate with the doctor's treatment on the basis of this persuasion.

Childhood anxiety state: ${ }^{11}$ The occurrence of anxiety disorders in children which happens from infants, young children to teenagers has a close relationship with children's environment besides genetic factors. These environmental factors mainly include family, school and social activities. Through these environmental factors are the most significant factors, especially for children with genetic susceptibility factors under the influence of adverse environmental factors. While we initiate the treatment with child psychotherapy, we also need to educate parents to help children overcome anxiety, encourage children to participate in sports activities. Collective activities help children to be sociable, feel less loneliness and is good for children to overcome shyness, timidity, autism and other weaknesses; Taking part in the co-curricular activities can help children to overcome from problems like easily irritable, emotional impulsive weakness and lack of self-control, etc.

Child autism: ${ }^{12}$ The typical clinical manifestations of children with autism are language development disorders, social interaction disabilities and repetitive behavior. Some children have different levels of developmental disorders. According to the general low prevalence estimates in China, ${ }^{13}$ there are about 100000 autism children. Autism is a chronic course disease, prognosis is connected with the severity of the disease, childhood language development situation, the discretion of the intelligence quotient (IQ), training, education and timeliness of disease intervention. Child care doctors provide professional knowledge, formal training and treatment at an early date.

In a word, China has the largest population in the world, the number of children and adolescents also reached more than 367 million, it is extremely urgent for us to pay attention to our child and adolescent mental health problems.

\section{REFERENCES}

1. Frank V. Commentary: Physical health outcomes and health care have improved so much, so why is child mental health getting worse? Or is it? A commentary on Collishaw. J Child Psychol Psychiatry 2015; 56(3):394-396.

2. Michael $H$, Heike $H$, Bärbel MK and Robert S. How often are German children and adolescents diagnosed with ADHD? Prevalence based on the judgment of health care professionals: Results of the German health and examination survey (KiGGS). European Child \& Adolescent Psychiatry 2008; 17(1): 52-58.

3. Zhang $Y$ and Liu SX. $4 \sim 16$ years old children's psychological behavior problems of epidemiological survey. China's maternal and child health care 2007; 13:1829-1830. (In Chinese).

4. Pan RD and Wu M. Children's psychological behavior problems epidemiological investigation in China. Journal of medical anthologies 2004; 01:74-76. (In Chinese).

5. Sun TT. Special family children's mental health and subjective well-being research. Liaoning: China medical university, 2010.

6. Xia F and Bai XP. Family environment factors to the analysis of preschool children's psychological behavior development. Journal of Hainan Medical 2005; 10: 142-144. (In Chinese).

7. Li ZKi. Study on the relationship between the resilience and social support of left-behind children. Chinese journal of health psychology 2009; 440-442.

8. Chen $X Z$. Child mental flexibility based on social cognitive research. Shanghai: East China normal university, 2006.

9. Zhen JJ. Centered on behavioral therapy treatment of children psychology case study. Guangzhou: South China normal university, 2005.

10. Hou ZJ. Children's development and current situation of the psychological counseling and treatment. Journal of capital normal university 1996; 126:126-132.

11. Zhou N. Theory of games in children's psychological and behavioral problems of treatment application. Science wenhui 2008; 11:48-49. (In Chinese).

12. Cheng $C$, Zhang ZX and Zhang CY. Painting therapy - the importance of promoting children's mental health method. Journal of mental health education of primary and secondary schools, 2011, 12, 14 and 16. (In Chinese).

13. Su CX, Wang $X Y$ and Li $Y X$. Mood disorders in children report 1 case of psychotherapy process. Journal of Chinese clinical rehabilitation 2005; 44:75-77. (In Chinese). 